\title{
The use of nanomodified heat storage materials for thermal stabilization in the engineering and aerospace industry as a solution for economy
}

\author{
Alexander Shchegolkov ${ }^{1, *}$, Aleksei Shchegolkov ${ }^{1}$, and Alevtina Demidova ${ }^{2}$ \\ ${ }^{1}$ Tambov State Technical University, 392000, Tambov, Russia \\ ${ }^{2}$ Peoples Friendship University of Russia , 117198, Moscow, Russian
}

\begin{abstract}
The paper presents studies on the use of nanomodified materials with phase transitions. Thermostable heat exchange devices based on materials modified with carbon nanotubes allowing to control thermal contact have been developed. It is established that through a controlled thermal contact it is possible to provide a heat flux of up to 100 $\left[\mathrm{kW} \cdot \mathrm{m}^{-2}\right]$. The application of heat-resistance storage material in technical devices such as self-rescuers is justified. The efficiency of thermal control is established, since the temperature after the heat exchanger is reduced to $40{ }^{\circ} \mathrm{C}$ at an incoming temperature of about $100{ }^{\circ} \mathrm{C}$.
\end{abstract}

\section{Introduction}

The sustainable development of industry in the 21-st century requires economic growth, as well as careful use of resources and environmental protection. New materials that can integrate into information technology (industry 4.0) are becoming a key factor for the innovative development of the manufacturing base in the engineering and aerospace industries. The demand for new materials that will exceed traditional materials is dramatically increasing. To obtain new types of materials, the technology of composites production is used. Composites have properties that are inaccessible to conventional materials. In addition to improving thermal properties, such as thermal conductivity, materials should retain functionality at much higher operating temperatures. And additionally provide new functionality related to the regulation of their physical parameters. In this case - the property of control of physical parameters allows to attribute new materials to the generation of "smart".

The use of smart materials with special thermal properties for thermostabilization in engineering and the space industry opens up a whole new set of possibilities. These include an increase in the efficiency of heat transfer at a much lower mass.

The main technological directions and introduction of heat storage materials include:

- thermal control of bearing units

- control of heat fluxes in various heat exchange elements

- thermal regulation with air cooling

\footnotetext{
*Corresponding author: Energynano@yandex.ru
} 
- thermal regulation of thermally loaded elements

To address these issues, it is necessary to conduct studies with special temperature operating conditions for different types of heat-storage materials, especially for those obtained by nanomodification. A study of a composite material with a phase transition effect (PCM) containing $\mathrm{Cu}(\mathrm{NP})$ nanoparticles was carried out in [1]. The results of XRD and TEM showed that $\mathrm{Cu}$ NP are pure metals with irregular morphology. The diameter of $\mathrm{Cu}$ NPs was in the range of 5-15 $\mathrm{nm}$. The material melted at a temperature of $310 \mathrm{~K}$, and the total enthalpy of the phase transition was $122\left[\mathrm{~J} \cdot \mathrm{g}^{-1}\right]$. The thermal conductivity of composite PCM decreased with the introduction of $\mathrm{Cu}$ NPs from $0.130\left[\mathrm{~W} \cdot \mathrm{mK}^{-1}\right]$ to 0.102 $\mathrm{W} / \mathrm{mK}$. The authors suggest that such material can be used for thermal protection. However, the improvement of thermal conductivity is more urgent than reducing it. In the paper [2], investigations were carried out to determine the influence of copper nanowires (CuNWs) and copper nanoparticles (CuNPs) on the thermal conductivity of dimethicone nanocomposites. CuNWs and CuNPs were obtained using the liquid phase reduction method. Experimental data show that the thermal conductivity of composites increases with increasing filler. With the addition of $10 \% \mathrm{CuNWs}$, the thermal conductivity of the composite is $0.41\left[\mathrm{~W} \cdot(\mathrm{m} \cdot \mathrm{K})^{-1}\right]$. The normalized thermal conductivity gain of 2.73 is much higher than that of an analog containing CuNP (1.67). These experimental data are consistent with the model prediction. The authors found that $1 \mathrm{D} \mathrm{CuNW}$ can create thermal networks more efficiently than CuNP in the composite, which leads to higher thermal conductivity [2]. Of considerable interest are studies to improve the properties of paraffins. It is established that when paraffins are filled with carbon nanotubes (CNTs), the thermal conductivity can increase up to $30 \%$ [4]. The introduction of montmorillonite into paraffin resulted in an increase in thermal conductivity of $38 \%$ and ensured the thermal stability of the composition for 100 charge-discharge cycles [3]. As a result of the joint use of montmorillonite and carbon nanotubes as modifying components, it was possible to increase the thermal conductivity of the initial paraffin matrix by $73 \%$ [3]. It was shown in [4] that the thermal conductivity (ie, the heat transfer rate) between the contacts of multilayered CNTs can increase up to 27 times due to an increase in their diameter of only $58 \mathrm{~nm}$. In the study of compositions containing carbon fibers, the authors of Ref. 5 indicate that a decrease in the number of contacts between particles with high conductivity should be minimized. Modern studies show an increase in the thermal conductivity of suspensions as the length of graphene flakes increases [6]. This effect is explained by the ballistic regime of thermal conductivity of low-frequency acoustic phonons [7]. Also in this paper, experimental results are presented, which demonstrate an increase in the thermal conductivity of paraffin by almost 2 orders of magnitude when using graphenes. The concentration of graphene in paraffin was $20 \%$ by mass, the thickness and length of the flakes were 1-8 $\mathrm{nm}$ and 500-1000 $\mathrm{nm}$, respectively. It should also be noted that the thermal conductivity strongly depends on the content of graphene in paraffin. The authors of [8] used 10 times less concentration in [7], the thermal conductivity of the nanocomposite increased by only $60 \%$. In an article from the practical application of modified paraffin wax for a lithium-ion battery, the introduction of $20 \%$ graphene can provide an increase in thermal conductivity of up to $50\left[\mathrm{~W} \cdot \mathrm{mK}^{-1}\right]$ while maintaining the possibility of heat accumulation on the phase transition effect [9]. However, the high cost of graphene leads to the need to look for materials that increase the level of thermal conductivity at lower concentrations and values. Such materials include graphenes with magnetic properties. The paper [10] shows studies of the thermal conductivity of materials with the effect of selfleveling in the structure of a modified material. The method of synthesis of nanocomposite graphene with $\mathrm{Fe}_{3} \mathrm{O}_{4}$ nanoparticles is presented. Self-leveling of "magnetic graphene" improves the thermal conductivity of composites and epoxy resins. Improvement of thermal conductivity with oriented fillers occurs at low concentrations of the order of $\sim 1 \%$. At the 
same time, the authors of [10] consider materials that do not possess the phase transition effect.

Actuality of the research: Ensuring the effective operation of a certain class of processes associated with ventilation or cyclic cooling, as well as heating and the need to realize a rapid accumulation of heat with time, with effective retention and subsequent release (discharge). Such processes include the processes of accumulation of thermal energy in solar collectors, air ventilation in heated rooms, bus and car salons, and self-rescuers with exothermic reactions.

\section{Objectives of the study:}

1. Investigation of methods for the modification of materials with phase transitions, carbon nanotubes.

2. Development of heat-stable heat-exchange devices based on materials modified with carbon nanotubes.

3. Development of heat storage materials controlled by a magnetic field.

4. Justification of the use of heat-resistant heat-accumulating material in technical devices.

\section{Methods and Materials}

Samples were prepared on the basis of composites containing paraffin P-2 (Russia), fluoroplastic (F-4MB). As a modifying additive, the multi-layer cylindrical CNT of the Taunit series (manufactured by Ltd. Nanotechcenter, Tambov, Russia) with an outside diameter 15-40 $\mathrm{nm}$ and an internal diameter of 3-6 nm (Figure 1). The method of CNT synthesis is described in [12 Nanotubes Taunit or Taunit-M (0.5-10\% by weight) CNT is mechanically activated by cyanide ferrite EE65B up to $5 \mu \mathrm{m}$ in a ball mill for 20 minutes. Modification of paraffins produced by CNT with the desired morphology will ensure interactions at the molecular level and create a stable material with the characteristics that are necessary for the formation of TM. The modification occurs during the phase transition of paraffin, when it passes into the liquid state at the temperature at which the threshold of its thermal decomposition is reached, which allows the reactivity of CNM with paraffin molecules to initiate this process. It is necessary to use ultrasound, which provides the phenomenon of cavitation in the volume of molten paraffin. To ensure the desired orientation of the structures integrated in the CNM, upon cooling, the molten paraffin is exposed to a magnetic field. After this, the material is subjected to mechanical action in the extruder using a fluoroplastic screw. The power of the drive motor of the extruder is $1 \mathrm{~kW}$ at a speed of $60 \mathrm{rpm}$. In addition, the resulting material is granulated in a compacting granulator to form granules to a size in the range of 1 to $2 \mathrm{~mm}$. As a result, the individual granules are spheres with a diameter of 1 to $2 \mathrm{~mm}$. After this operation, the granules are filled with a carrier fluid.

To obtain a heat-exchanging element, a method for obtaining a heat-absorbing material by applying heat-absorbing paste onto a fibrous material substrate is used, by preparing a dispersion system in which the dispersed phase is carbon nanotubes (CNTs) and the dispersed medium is a molten mixture of paraffins, cooling the disperse system to produce a paste viscosity, characterized in that polyethylene wax is introduced into the heatabsorbing paste in an amount of wt. $\% 5$ to 6 , the heat-absorbing paste is applied by rubbing the paste into the recesses of the non-woven polymeric material backing before the paste appears on the back side of the substrate and a continuous coating is obtained on the front side.

To study the distribution of the temperature field on the surface of the heat exchange equipment, a contactless method for measuring the temperature field was used. The device 
in which the presented method is implemented is a thermal imager. The following equipment was used in the research: the Thermo-Imager Testo 871-1.

\section{Results and discussion}

Fig. 1 shows the behavior of the nanomodified heat storage material (TM) distributed in a liquid carrier under the action of a magnetic field created by a permanent magnet (1000 $\mathrm{mT})$.

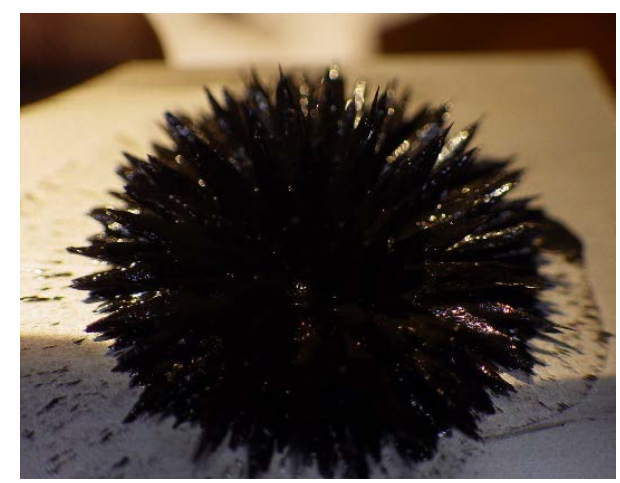

Fig. 1. Nanomodified TM under the action of a magnetic field

Figure 2-3 shows the heat transfer process in TM in the form of a thermogram under conditions of the action of a magnetic field $(1000 \mathrm{mT})$. Figures 3-4 show the possibility of controllability of heat exchange processes and, in particular, thermal contact. The influence of the magnetic field leads to the formation of "cones", which are shown in Figure 1. In this case, the "cones" begin to touch the opposite surface, and through them the heat flow is transmitted, which is directly proportional to the contact area, thermal resistance and temperature.

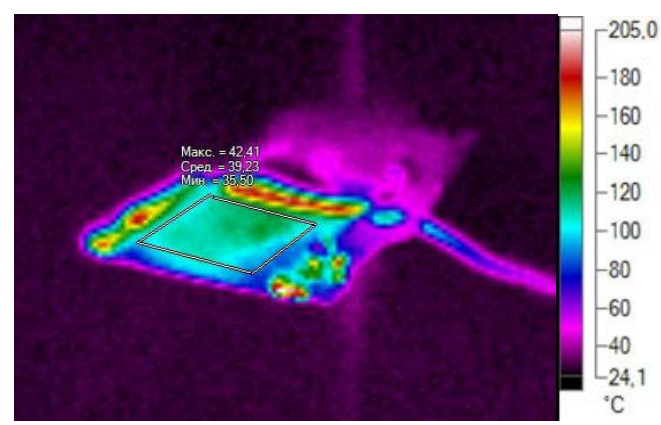

Fig. 2. Heat exchange platform with TM under magnetic field conditions 


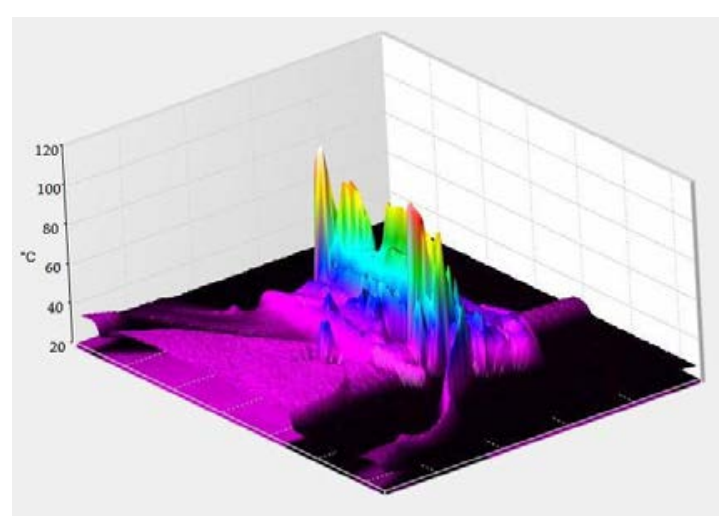

Fig. 3. 3D thermogram of a controlled thermal contact

Figure 4 shows the thermogram of the heat storage material under the action of hightemperature action.

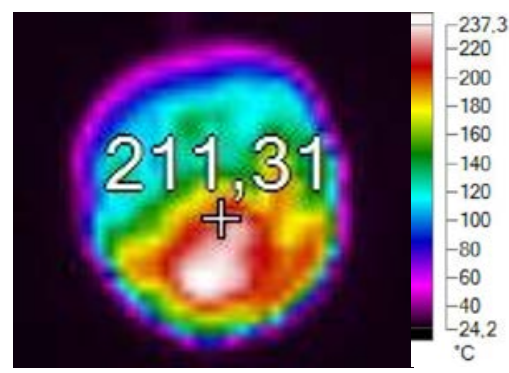

Fig. 4. Thermogram of TM under the action of high temperatures

The thermal stability of TM is established under the influence of high temperatures - the material does not destroy. Figure 5 presents a 3D thermogram of the heat storage material under the action of high-temperature action.

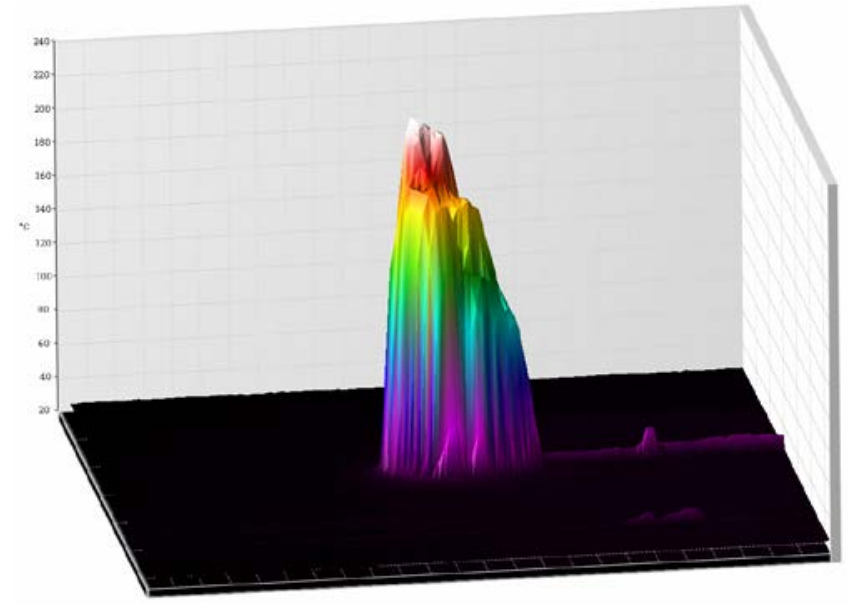

Fig. 5. 3D thermogram of of TM under the action of high temperatures

Fig. 6 shows the heat exchange in the thermal control system of a self-rescuer with a heat exchange element based on nanomodified paraffin [11]. The efficiency of thermal 
regulation is established, since the temperature after the heat exchanger is reduced to $40^{\circ} \mathrm{C}$ at the incoming temperature of about $100^{\circ} \mathrm{C}$.

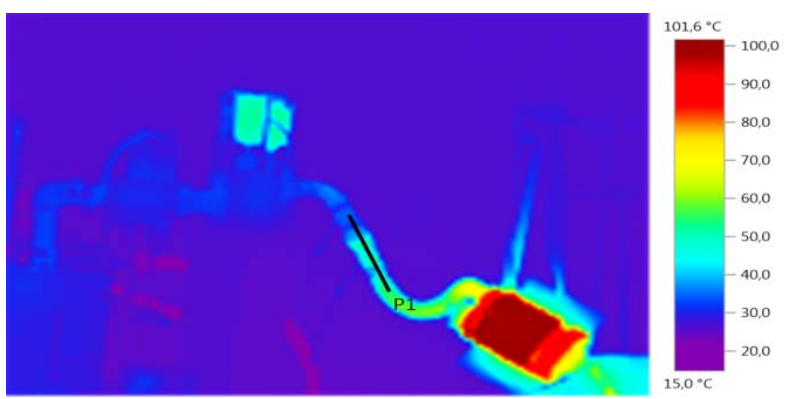

Fig. 6. Thermogram of self-rescuer - tests on the stand of artificial lungs

\section{Conclusions}

1. Investigations of methods for the modification of materials with phase transitions carbon nanotubes.

2. Thermostable heat exchange devices based on materials modified with carbon nanotubes allowing to control thermal contact have been developed. It is established that a heat flux of up to $100\left[\mathrm{~kW} \cdot \mathrm{m}^{-2}\right]$ can be provided through a controlled thermal contact.

3. Developed heat storage materials controlled by a magnetic field $(1000 \mathrm{mT})$.

4. The use of heat-resistant storage material in technical devices, such as self-rescuers, is justified. The efficiency of thermal regulation is established, since the temperature after the heat exchanger is reduced to $40^{\circ} \mathrm{C}$ at the incoming temperature of about $100^{\circ} \mathrm{C}$.

This paper was financially supported by the Ministry of Education and Science of the Russian Federation on the Program to improve the competitiveness of RUDN "5-100" University among the world's leading research and education centers in 2016-2020.

\section{References}

1. Ju-Lan Zeng, Ting Xie, Yu-Xiang Zhang, Liu-Mo Jiang, Kun-Yu Li, Sai-Bo Yu \& Shao-Fen Wang Energy sources, part a: recovery, utilization, and environmental effects, 1091 (2018)

2. Dahai Zhu, Wei Yu, Haixu Du, Lifei Chen, Yang Li,and Huaqing Xie Journal of Nanomaterials, 6 (2016)

3. M. Li, Q. Guo, S. Nutt Solar energy, 146 (2017)

4. Warzoha R. J., Fleischer A. S. Applied Energy, 154 (2015)

5. Fan L., Khodadadi J.M. Renewable and sustainable energy reviews, 15 (2011)

6. Xu X., L. Pereira F.C., Wang Y., Wu J., Zhang K., Zhao X., Bae S., Bui C.T., Xie R., Thong J.T.L., Hong B.H., Loh K.P., Donadio D., Li B., Özyilmaz B. Nature communication, 5 (2014)

7. Renteria J.D., Nika D.L., Balandin A.A. Applied sciences, 4 (2014)

8. Liu X., Rao Z Thermochimica acta, 647 (2017)

9. P. Goli, J. Power Sources, 248 (2014)

10. J. Renteria, et al., Materials and Design, 88 (2015)

11. A. V. Shchegolkov, Nano Hybrids and Composites, 13 (2017) 